\title{
Close encounters with scientific analogies of the third kind
}

\author{
Francesco $\mathrm{Nappo}^{1}{ }^{1}$
}

Received: 30 October 2020 / Accepted: 17 June 2021 / Published online: 30 July 2021

(c) The Author(s) 2021

\begin{abstract}
Arguments from non-causal analogy form a distinctive class of analogical arguments in science not recognized in authoritative classifications by, e.g., Hesse (1963) and Bartha (2009). In this paper, I illustrate this novel class of scientific analogies by means of historical examples from physics, biology and economics, at the same time emphasizing their broader significance for contemporary debates in epistemology.
\end{abstract}

Keywords Analogical Reasoning · Mary Hesse $\cdot$ Paul Bartha $\cdot$ Scientific methodology $\cdot$ Natural kinds

\section{Introduction}

Arguments from the observed properties of a scientific model to the unobserved properties of a target typically rely on analogical reasoning: often those arguments are accepted, rejected or considered stronger than others depending on the number and kinds of relevant similarities and dissimilarities existing between model and target (Bartha, 2009; Bayler-Jones, 2013; Hesse, 1963). We feel more confident that a vaccine will be effective on human beings after testing it on mice than after testing it on lizards; and more confident that a social policy will be effective in Los Angeles after obtaining positive results in San Diego than in Paris. This is because mice are more similar to humans than lizards; San Diego resembles Los Angeles more than Paris does.

The use of analogical reasoning as a form of inductive inference about the unobserved can be distinguished from at least one other instance of this reasoning in science. This is its role in the context of scientific illustration. An example is J. C. Maxwell's (1890) comparison between the motion of molecules in a gas and that of a swarm of bees (cf. Achinstein, 2013:138). In these cases, one appeals to analogical

Francesco Nappo

fr.nappo@gmail.com; francesco.nappo@polimi.it

1 Department of Mathematics, Politecnico di Milano, Via Edoardo Bonardi, 9, Milano 20133, Italy 
reasoning as a means of illustrating a concept or theory to an audience rather than as a basis for inferences about the yet unknown. Although the illustrative role of analogy is at least as important to scientific advancement as the inferential, the focus of this paper will be exclusively on the different ways analogies can fulfill the inferential role; the philosophical issues that pertain to the illustrative use require a separate discussion.

One way of classifying analogical inferences from scientific models is not according to their strength, but according to the analogies by which they are fueled. According to the taxonomy introduced by Hesse (1963), one ought to distinguish between arguments from material and from formal analogy (cf. Achinstein, 1964; BaylerJones, 2013; Pietsch, 2019; Fraser, 2019). On Hesse's view, the former class includes any argument that (a) draws upon 'material' or 'pre-theoretic' similarities between model and target, i.e., resemblances in scientifically respectable properties that can be recognized before and independently of the analogical argument ${ }^{1}$; and where (b) it is reasonable to expect that causal relations of the same kind as the ones that hold among the salient properties of the model also obtain among the corresponding properties of the target. ${ }^{2}$ A plausible example of a material analogical argument is when we reason from the effects of a vaccine on mice to its similar effects on humans, expecting that mice will resemble humans in precisely those features (of, e.g., the immune system) that are causally connected to mice's response to the drug.

For Hesse, any analogical argument that does not satisfy conditions (a) and (b) can be seen as falling into the class of arguments from formal analogy. ${ }^{3}$ In those cases, source and target are known to be linked by an isomorphism, i.e., a one-toone correspondence between the terms of each domain, but with no resemblances between those terms or between the relations that hold in each domain, except from those introduced by the isomorphism itself. ${ }^{4}$ The analogy between heat

\footnotetext{
1 Some authors are misled by Hesse's 'material' to suppose that source and target must have some 'physical' property in common for there to be material analogy (see, e.g., Dardashti et al., 2019:2 "[the analogy] is not material in Hesse's sense since there is no physical property in common"); but this is not necessary: see, e.g., Hesse's (1963:32) discussion on material analogies between light and sound. Hesse's view is that a similarity with respect to $\mathrm{P}$ counts as 'material' or 'pre-theoretic' at time $t$ when $\mathrm{P}$ belongs to the accepted vocabulary of some contextually relevant language community at $t$ (cf. 1963:15). Her condition thus serves to rule out respects of similarity that an analogical argument may introduce but that are not already accepted in the language - including, but not limited to, the similarities in 'gruesome' respects noted by Goodman (1955).

2 On the notion of a 'causal' connection, cf. Hesse (1963): " "causal' in the sense in which the relation of 'atmosphere' to 'humans' or 'wave-motion' to 'reflection' is causal' (77). Below I will explicate this in terms of co-occurrences mediated by causal laws. Cf. also Pietsch (2019) who invokes a manipulationist account.

3 The distinction between material and formal analogies can be regarded as exhaustive on Hesse's classification since, for any two domains not linked by material analogy, there is an arbitrary isomorphism between the properties of model and target that satisfies the definition of formal analogy. Cf. Hesse (1963): "any sufficiently rich theory could be made isomorphic to any given accepted statement" (49). Hesse accordingly regards the material/formal distinction as the "crucial" (1963:100) one for classification.

${ }^{4}$ Cf. Hesse (1963) on arguments based on the analogy of father-son with state-citizen, where there is no "horizontal relation of similarity..., except in virtue of the fact that the two pairs are related by the same vertical relation" (63). In this case, the similarities do not exist 'before and independently' but are introduced with the argument by way of making salient the vertical relations 'protector-of', 'obedient-to', etc.
} 
and electricity is a plausible example: there exists a one-to-one correspondence between the quantities related to heat (source of heat, flow, temperature, etc.) and electrical ones (center of attraction, field, voltage, etc.); however, the corresponding terms in the two domains are not similar: heat flow is unlike electric fields, temperature is unlike voltage, etc. What makes those different quantities 'analogous' to one another is solely the fact that (as it turns out) they play structurally similar roles in their respective theories. Hence, an inference that draws upon an isomorphism with heat to support a conclusion about electricity is one from formal analogy.

We can rephrase the distinction between material and formal analogies by adopting the following schematic representation for analogical arguments, due to Hesse (1963:60):

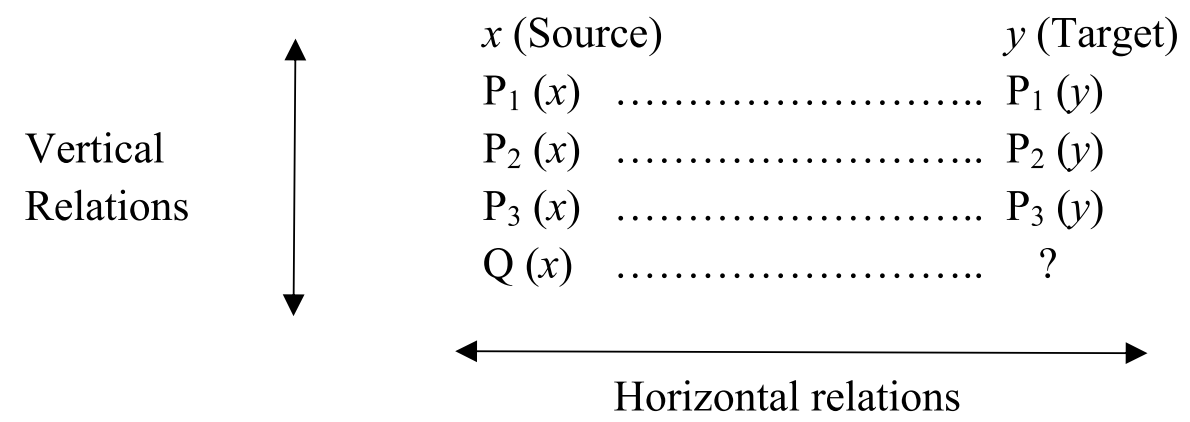

In this representation, the ' $\mathrm{P}_{\mathrm{n}}$ ' in each domain stand for the properties that figure in the premises of the analogical argument - i.e., the similarities that are used as evidence in the argument; 'Q', instead, stands for the respect of similarity which figures in the argument's conclusion.

According to Hesse, in arguments from material analogy the so-called 'horizontal relations' connecting the observed properties $\mathrm{P}_{\mathrm{n}}(x)$ of the source and the corresponding $\mathrm{P}_{\mathrm{n}}(y)$ observed in the target are relations of material similarity; the socalled 'vertical relations' connecting the $\mathrm{P}_{\mathrm{n}}(y)$ with some yet unobserved $\mathrm{Q}(y)$ in the target are expected to be causal relations of the same kind as those that connect the $\mathrm{P}_{\mathrm{n}}(x)$ with $\mathrm{Q}(x)$ in the source. These two features correspond, respectively, to the satisfaction of conditions (a) and (b). In arguments from formal analogy, the 'horizontal relations' are correspondences according to some one-to-one mapping of the $\mathrm{P}_{\mathrm{n}}(x)$ to the $\mathrm{P}_{\mathrm{n}}(y)$, whereas the 'vertical relations' can be any relations, whether causal or of some other nature, that connect the terms in the respective theories of $x$ and $y$ (cf. Hesse, 1963:69). ${ }^{5}$ Clearly, this means that 'material analogy' and 'formal analogy' in Hesse's sense are mutually exclusive.

\footnotetext{
${ }^{5}$ I should emphasize that this notion of formal analogy is different and more restrictive than the ones employed by Bartha (2009) and Norton (2020) On Bartha's notion, see fn.14. On Norton's (2020) notion, see his quote: "the fertile analogy between Newtonian gravity and Coulomb electrostatics... is a formal analogy in that it connects gravitational and electrostatic fields by virtue of their both satisfying the same field law" (11). Even though this is a notion of formal analogy, it is not the notion that Hesse (1963) and other authors employ.
} 
This paper aims to show, by means of historical examples, that this influential classification misses an important category of arguments from analogy in science: arguments from 'non-causal analogy'. 6 Those arguments draw upon genuine similarities (as opposed to pure isomorphisms) between a model and a target; at the same time, the salient vertical relations are connections modally stronger than causation - typically, they are deductive entailments mediated by some logico-mathematical theorem. Because of these features, a broadening of Hesse's category of material analogy is required to include them. That is, the class of arguments from material analogy should be revised so as to include both: (i) arguments that draw upon material similarities and where the vertical relations in each domain are causal (arguments from 'material analogy' in Hesse's original sense), and (ii) arguments that draw upon material similarities and where the vertical relations are distinctly non-causal - 'arguments from non-causal analogy'.

The case-studies that I will discuss not only require a revision of Hesse's (1963) taxonomy, but also pose a problem for more recent classifications. I will illustrate this point by showing that arguments from non-causal analogy are not accounted for by an alternative taxonomy of analogical arguments in science, defended by Bartha $(2009,2019)$. This alternative classification assigns practically no significance to the nature of the horizontal relations in the analogical argument and relies almost exclusively on the nature of the vertical relations that are made salient by the analogy. As will be discussed, the case-studies presented in this paper fall in between Bartha's "mathematical" and "abductive" (2009:99) analogies and require a new partition of his alternative classification as well. This is further evidence that non-causal analogical arguments are not instances of some already known class of inferences.

The discussion will proceed as follows. In section two, I will present three case-studies of arguments from non-causal analogy respectively derived from physics, biology and economics, which illustrate that the traditional classification of arguments from analogy in science is not exhaustive. In sections three, I will consider two responses on behalf of the traditional account: the former aims to show that the case-studies are arguments from formal analogy; the latter, that they are simply other arguments from material analogy in Hesse's original sense. Having resisted those proposals, in section four I will move on to argue that the same three case-studies require a modification of Bartha's (2009) taxonomy of analogical arguments in science. In section five, I will briefly discuss how the recognition of non-causal analogies bears on current debates in epistemology, including Norton's (2020) argument to the effect that no general schema can be devised by which we can assess the strength and quality of analogical arguments in science.

\footnotetext{
${ }^{6}$ This terminology is borrowed from the literature on non-causal explanations in science (e.g., Lange 2016).
} 


\section{Case-Studies}

In the following sub-sections, I will present three historical examples of arguments from analogy having the feature that $(i)$ they are used inferentially, i.e., to support the conclusion that a non-trivial property $\mathrm{Q}$ that holds for the model also holds for the target ${ }^{7}$; (ii) they do so by drawing on material similarities $\mathrm{P}_{1} \mathrm{P}_{2}, \mathrm{P}_{3}, \ldots$ between model and target; but where (iii) given the background knowledge, there is no reasonable expectation that roughly the same kinds of causal connections that obtain between $\mathrm{P}_{1} \mathrm{P}_{2}, \mathrm{P}_{3}, \ldots$ and $\mathrm{Q}$ in the model will also obtain in the target. ${ }^{8}$ Even though the notion of a 'causal connection' is vague, in what follows I will take it to refer generally to any tendency to co-occur that is mediated by causal laws, i.e., by empirical generalizations connecting causes to their effects. ${ }^{9}$ The examples below will clarify the intended contrast.

Heisenberg's Symmetries Relying on the known resemblances between, on the one hand, pairs of protons and neutrons inside the nucleus and, on the other hand, pairs of electrons outside, Heisenberg (1932) argued that protons and neutrons are united by a form of invariance under SU(2) transformations analogous to the invariance satisfied by pairs of electrons in, respectively, 'spin-up' and 'spin-down' mode. In other words, just like two electrons observed respectively in 'spin-up' and 'spin-down' are not two different entities, but simply electrons in different modes, so for Heisenberg protons and neutrons are the same particle, the 'nucleon', but with different 'spin' properties - or analogues thereof. As physicists today would put it, to remark on the analogy (rather than the identity) with electronic spin, protons and neutrons possess different 'isospin'. The term 'isospin' is justified partly by the fact that whereas electronic spin is defined in (real) three-dimensional space, isospin is defined over a fictitious multi-dimensional space (cf. Steiner, 1998:87-89); therefore, even though the symmetry is the same mathematically, it differs from spin in that it is not recoverable in the three dimensions.

\footnotetext{
${ }^{7}$ Material non-causal analogies employed in a non-inferential way are well-documented in the literature. See, e.g., Achinstein (2013:133) on Maxwell's fluid analogy to electricity in "On Faraday's Lines of Force" (1856). I am including in the 'inferential' category the so-called heuristic use of analogy as a device of discovery.

${ }^{8}$ Other scholars have hit upon arguments satisfying these conditions, but without recognizing them as forming a sui generis class of analogical arguments: see, e.g., Pincock (2012) on models of gregarious behavior.

9 This understanding of 'causal connection' is meant to be an especially liberal one as it includes analogical arguments in which the salient vertical relations are not relations of cause-effect, but of entailment mediated by a causal law. Cf. the examples of Priestley's and Stokes' analogical arguments mentioned in section four.
} 
The schematic representation invoked in section one helps us see precisely what makes Heisenberg's analogy non-causal. ${ }^{10}$ Here is a simple way of representing the analogy:

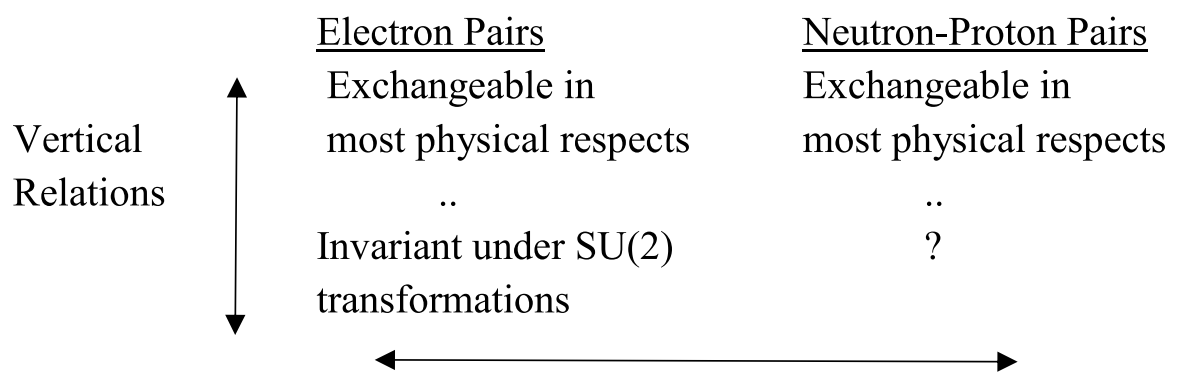

Horizontal relations

As the schematic representation illustrates, the main similarity that inspired the analogy with alternatively spinning electron pairs is the fact that proton-neutron pairs are exchangeable in most physical respects (besides charge): for instance, they have roughly identical mass and behave in the same way with respect to the strong force (cf. French, 2000). Heisenberg was also aware of the other analogies between atomic and nuclear physics (omitted in the schematic representation) that past physicists had built upon in order to tackle the difficult questions regarding the composition of atomic nuclei (cf. Darrigol, 1988). Altogether, these facts are evidence that the analogical argument draws upon material similarities in Hesse's sense, i.e., that the similarities that figure in the premises can be recognized as genuine even before the argument is introduced (and independently of the 'vertical relations' made salient by it).

But while the horizontal relations in the analogical argument are material similarities, the vertical relations are not causal. The reason lies with the distinction that many physicists and philosophers draw between causal laws and constraints (cf. Lange, 2016, chapter 3 and references therein). The exchangeability properties of electrons and their invariance under SU(2) transformations are related to one another: from knowing their SU(2) symmetry, we can deduce that an electron in spin-down is exchangeable in most physical respects with an electron in spin-up after a 360 degrees rotation; and also that, at a second attempt at rotation, each electron needs to be turned 720 degrees to go back to its initial state. However, these and similar observable consequences do not hold in virtue of some causal law that alternatively spinning electron pairs obey. Rather, the SU(2) symmetries are more akin to space-time symmetries: they are physical constraints on the possible causal interactions in a given domain. It is arguably this non-causal constraining of the properties of electron pairs by the underlying SU(2) symmetry that the argument projects onto

\footnotetext{
${ }^{10}$ Steiner (1998) regards this as a case of formal analogy. As I am about to explain, however, it is implausible to reduce the similarities in Heisenberg's argument to merely structural correspondences (cf. French 2000).
} 
neutron-proton pairs. In an interview, Heisenberg himself noted: "I suspect isospin is a symmetry similar to space and time" (in Buckley \& Peat, 1996:14).

Admittedly, not everyone accepts the distinction between causal laws and constraints that I am invoking. But I also have little to add to what has already been said in its defense (e.g., Lange, 2016). To side-step this difficulty, let's consider two more cases of arguments from non-causal analogy in science whose recognition does not rely on that debated but plausible distinction (Fig. 1).

Galton's Statistical Identities Galton's “Typical Laws of Heredity” (1877) addresses a puzzle about biological populations. According to the data collected by Quetelet with regards to the inheritable traits in various biological populations, different generations of the same population often exhibit 'statistical identity': barring significant changes in environment, the mean and deviation of the distribution of any heritable trait in each generation of a population is almost exactly the same as the mean and deviation of the distribution in any previous or later generation. To explain this surprising phenomenon, Galton (1877) offered an analogy with a mechanical apparatus called the 'quincunx' (Fig. 1). In short, Galton showed that if on the top of a shot-dropping machine that produces bell-shaped distributions of pellets one places another machine that operates as a 'counterbalance' of the subsequent dispersion,

Fig. 1 Galton's Quincunx.

Visualize the machine as having two equal parts and three compartments. Top half: Pellets are distributed in vertical compartments so as to form a bellshaped curve. The pellets then fall through shoots (the oblique lines) possessing a specific angle of inclination, forming a narrower and taller bell-shaped curve. Bottom half of the machine: the pellets collected in the middle compartments are made to fall through a series of spikes (arranged in such a way that, for any spike encountered, there is the same chance that the pellet will fall to the right as to the left of it). As the result of this setup (especially the angle of inclination of the shoots, which measures the strength of reversion) the mean and deviation of the curves at the top and bottom compartments tend invariably to be the same

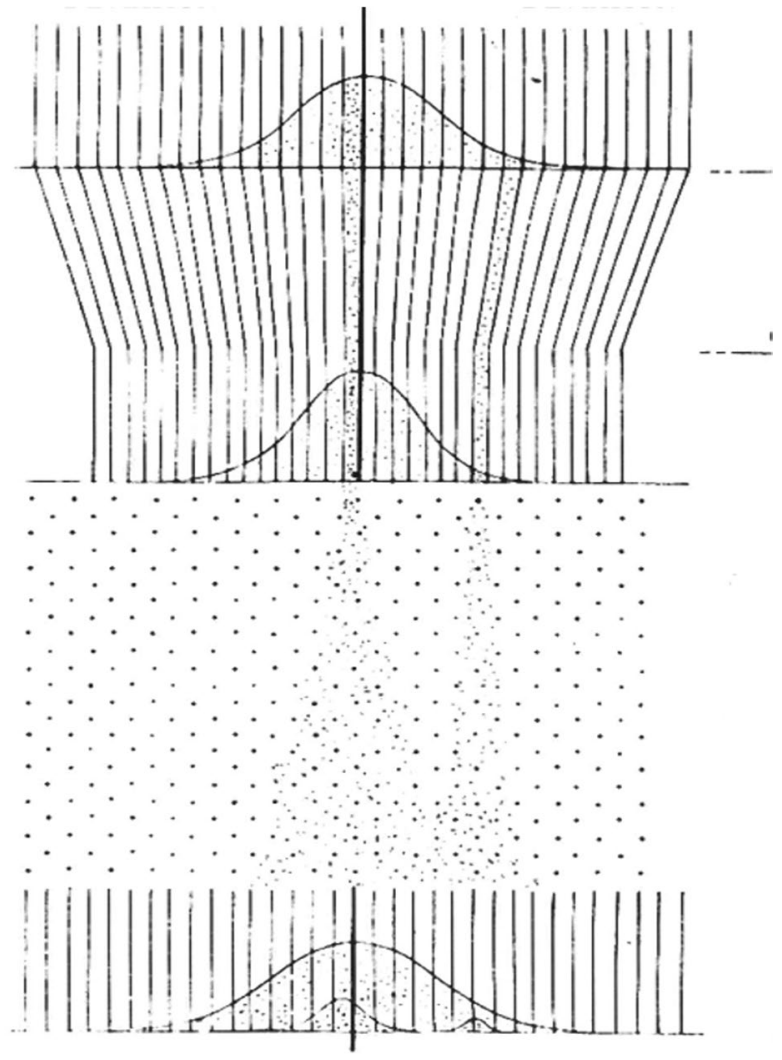


whose specific strength is in linear proportion to the amount of dispersion, then the resulting two-piece machine will systematically replicate occurrences of statistical identity. Galton used this observation to support his claim that a process of 'reversion' generally occurs in inheritance to balance the dispersive effect of genetic variation, in this way supporting an explanation of the otherwise mysterious occurrence of statistical identity in real-world populations of plants and animals.

Galton's argument for the presence of 'reversion' in population biology (obeying a law of linear proportion to the amount of dispersion) is another example of an argument from a non- causal analogy. Once again, a schematic representation of the analogy is helpful:

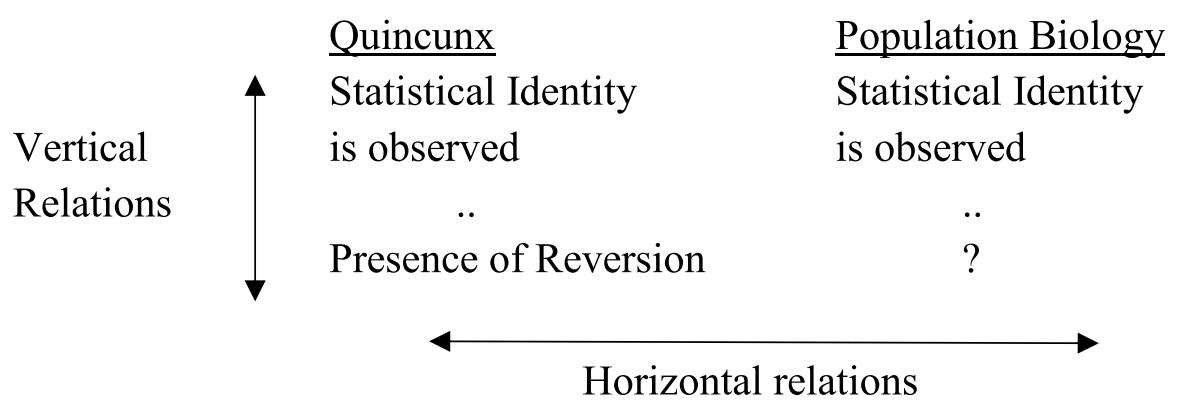

On the one hand, there are material resemblances between source and target of the analogy: in particular, there are obvious similarities between occurrences of statistical identity that the quincunx invariably produces and the ones observed in the population data. Such similarities can be recognized quite independently of the vertical relations made salient by the argument and hence are not merely formal. On the other hand, the vertical relation holding between the instantiation of statistical identity and the law of reversion is not causal. The connection that links reversion to the occurrence of statistical identity is entailment mediated by a theorem of statistics: statistical identity follows from the assumption that a reversion effect in linear proportion to the amount of dispersion obtains in the passage from one generation to the next. ${ }^{11}$ It is this non-causal connection that Galton's argument projects from the quincunx apparatus onto population biology, making his argument for reversion based on non-causal analogy.

Schelling's Segregation Patterns Whereas segregation in cities was often explained by racism, Schelling (1978) proposed a new model to show that segregation could be sometimes the consequence of a less problematic factor: a mild preference not to be a minority group among one's neighbors. Using dimes and pennies randomly

\footnotetext{
${ }^{11}$ Cf. also Hacking (1990), according to which Galton's discovery that statistical identity in population biology might possess an explanation in terms of an "autonomous statistical law" (186) sanctions the "taming of chance" (180). The same example is discussed in richer historical detail in Stigler (2016) and Nappo (2020).
} 
distributed over a checkerboard, Schelling showed that a form of segregation among dimes and pennies is frequently generated by repeated applications of the following rule: whenever a token (dime or penny) has less than one third of its neighbors of its own type, move it to the nearest empty space where at least one-third of its neighbors are of the same type. The claim about segregating patterns in cities that the checkerboard model supports is that, at least in some cases in which the hypothesis of racism had been regarded as the best explanation of segregation patterns, the observed segregation may instead possess an explanation in terms of a preference not to be a minority (as exemplified by the rule to move a dime or a penny when less than one third of its neighbors are its likes).

Schelling's argument about segregating patterns in cities is another plausible instance of an argument from a non-causal analogy. Schematically, the analogy can be represented as follows:

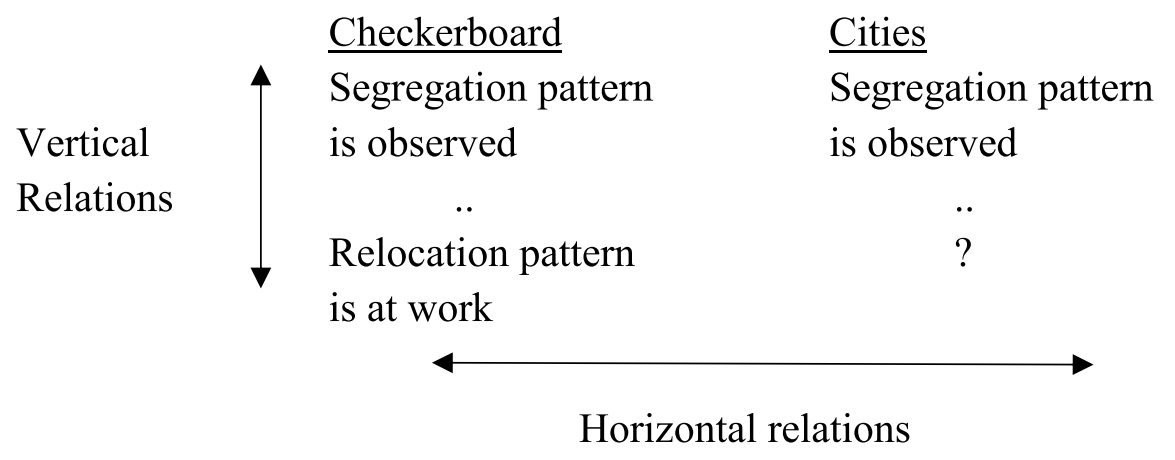

On the one hand, the checkerboard model resembles actual cities in various nonformal respects: for instance, in the division into neighborhoods, the possibility of relocating elsewhere, etc. ${ }^{12}$ On the other hand, the argument does not presuppose that the same causal connections that obtain in the checkerboard model are also likely to obtain in actual cities: among other things, dimes and pennies do not have 'preferences', they do not 'choose' to relocate, and so on. Rather, as Sugden (2000) has noted, the tendency of Schelling's rule to generate the segregation outcome in the checkerboard is the result of a purely mathematical necessity: given the checkerboard setup, it follows that the segregation outcome will occur frequently. As Sugden (2000) puts it, "That is not an empirical claim at all: it is a theorem" (17). It is this entailment relation between the checkerboard's setup and its segregation outcome, rather than any causal connection between the two, that the argument projects onto real-world cities. Because the horizontal relations are material similarities but the vertical relations are non-causal, the analogy is non-causal.

\footnotetext{
12 Grüne-Yanoff (2013) claims that there are no similarities between segregation in the checkerboard and in actual cities. However, this is implausible. Cf. also Fumagalli (2015) for a critique of Grüne-Yanoff's claim.
} 


\section{Why Non-Causal?}

In the previous section, I have discussed three historical examples of analogical arguments in science that satisfy neither Hesse's definition of inferences from formal analogy nor her definition of inferences from material analogy. In my view, a broadening of Hesse's class of arguments from material analogy is needed to include these case-studies. The common characteristic of 'material' analogical arguments in this broadened sense would be that the horizontal relations in the analogy are material similarities; those arguments can then be further partitioned into two categories - what we might call arguments 'from causal' and 'from non-causal analogy'- depending on the specific nature of the vertical relations involved.

As a way of defending the distinctiveness of the case-studies from section two, in the next sub-sections I will consider two potential objections. One aims to show that the case-studies of section two are merely other arguments from formal analogy, while the other that they are merely other arguments from material analogy in Hesse's (1963) original sense - i.e., material analogical arguments of the ordinary 'causal' variety. I will argue that neither of these attempts at rescuing the traditional bi-partition of analogical arguments in science is successful.

If It's Non-Causal Then It's Formal According to my reconstruction, the arguments presented in section two are not plausibly classified as based on formal analogy in Hesse's sense. In the latter case, one reasons to a particular conclusion about a target from knowing a mapping between the quantities figuring in a theory about the target and those belonging to some familiar source. For instance, from knowing that the equations of heat and electricity are formally the same, one might infer that the quantity of electric flow depends, like heat flow, on the thickness of the insulator multiplied by a constant $k$. Whatever we think about the status of such inferences, they are not a fitting category for the analogical arguments of section two: for instance, as previously discussed, Galton's argument for the reversion law starts off from material resemblances between the curves observed in the population data and those that his quincunx tends to produce. This feature makes the case-studies more like Hesse's arguments from material analogy, drawing upon material similarities, than her arguments from formal analogy.

Someone might object to this claim that the assumed notion of 'argument from formal analogy' is too narrow. ${ }^{13}$ One might, for instance, want to include in that category not only arguments that draw upon, but also those that conclude to, a formal resemblance in the quantities of two domains (as exemplified by cases in which a common equation links two distinct physical domains). ${ }^{14}$ In other words, one may regard as 'from formal analogy' not only those inferences in which an isomorphism figures in the premises $\left(\mathrm{P}_{1}, \mathrm{P}_{2}, \mathrm{P}_{3}\right)$ of the argument (as in Hesse's account), but also those in which an isomorphism appears in the conclusion (Q). This broader

\footnotetext{
13 I am extremely grateful to an anonymous referee for insightful suggestions on this specific response.

14 Cf. Bartha (2009): "we have a formal analogy between two domains whenever we can abstract a common mathematical form... regardless of whether this relates laws or descriptions of empirical phenomena" (210).
} 
definition fits the case-studies of section two: Heisenberg's argument, for instance, concludes that a common equation links the spin of electron pairs to the predicted iso-spin of neutron-proton pairs, in that they are shown to satisfy mathematically the same symmetry; similarly, Galton's argument concludes that heredity in biological populations is subject to the same statistical laws as the distribution of pellets instantiated in the quincunx apparatus.

While a legitimate notion of 'argument from formal analogy', the liberal construal arguably clashes with the spirit behind Hesse's classification: as Hesse (1963:39; cf. Achinstein, 1964: 328) herself noted, resemblances in mathematical form between any two domains are very easy to find. By adopting a liberal definition, then, a defender of Hesse's classification would face a trivialization problem: for any analogical argument can be understood as an argument 'from formal analogy' as long as an arbitrary isomorphism links source and target. In effect, a liberal construal of 'arguments from formal analogy' is more common among those who deny that Hesse's distinction is epistemologically significant (e.g., Bartha, 2009; Norton, 2020).

The conclusion that I mean to draw is that, if one thinks that there is something to Hesse's classification, and specifically to her distinction between material and formal analogical arguments, then one ought to keep her narrow construal of 'argument from formal analogy'; accordingly, one must classify the arguments of section two as a novel kind of argument from material analogy within Hesse's taxonomy. This limited conclusion is all we need for the purposes of this sub-section. In section four, I will consider an alternative taxonomy, Bartha's (2009), that does away with Hesse's distinction between arguments based on 'material' and 'formal' similarities and embraces a liberal construal of 'argument from formal analogy'. In that context, I will show that those of section two are very unlike the 'arguments from formal analogy' that Bartha discusses. It follows that, even if one adopts a different taxonomy and a liberal construal of 'argument from formal analogy', the central conclusion of this paper still holds: the case-studies of section two constitute a novel class of analogical arguments in science yet unrecognized in the literature. ${ }^{15}$ Before we move on to Bartha's alternative taxonomy, however, let's consider a second response on behalf of Hesse's original classification.

If It Confirms Then It's Causal As we are still considering the possibility of rescuing Hesse's taxonomy, could it be argued that the arguments of section two are merely other examples of arguments from material analogy in her original sense, i.e., arguments where the horizontal relations are material similarities and the vertical ones are causal relations? As background for this discussion, let me be as clear as I can as to why the scientific analogies of section two deserve their own special class. On my account, what makes the scientific analogies of section two special is both that (a)

\footnotetext{
15 In effect, the analogical arguments of section two are a novel kind of argument 'from material analogy' in Hesse's taxonomy and a novel kind of argument 'from formal analogy' in Bartha's taxonomy. These claims are compatible so long as we keep in mind the terminological differences between Hesse and Bartha.
} 
the vertical relation between the properties $\mathrm{P}_{1}, \mathrm{P}_{2}, \mathrm{P}_{3}$ and the predicted resemblance $\mathrm{Q}$ is one of entailment and not cause-effect and (b) the entailment is mediated by various kinds of constraints that go beyond the ordinary causal laws; these can be physical constraints such as symmetries or conservation principles (as in Heisenberg's example) or constraints of the logico-mathematical kind (such as the mathematical theorems invoked in Galton's and Schelling's examples). The combination of considerations (a) and (b) is what make it especially implausible to think that the vertical relations in the analogy are causal.

Nevertheless, somebody might resist this conclusion. One prominent worry starts off from the observation that, from an epistemological standpoint, the arguments presented in section two appear to have an inductive, and not merely heuristic, use in science; in this sense, they resemble many arguments from material analogy in Hesse's original sense. For instance, by showing that recurrent statistical identity outcomes can be obtained in a domain as simple as that of pellets, Galton's analogy with the quincunx shows that appeals to a divine interest for species stability or systematic faults in data collection are neither sensible nor necessary to explain the surprising occurrence of statistical identity in population biology. At the very least, then, the comparison with the quincunx disconfirms some of the rival explanations of statistical identity in population biology. Because the analogy plausibly fulfills an inductive role, a defender of Hesse's account might insist that it is simply a material analogy in Hesse's original sense. After all, if the epistemological profile is the same, what important distinction is achieved by calling those connections 'noncausal'? Can we not make this simpler by calling them 'causal'? ${ }^{16}$

While again fully conceding that such stipulations are possible, let me mention two reasons why they are undesirable in the specific epistemological arena with which this paper engages. First, the distinctions that are being called into question between, e.g., causal laws, constraints, and mathematical theorems, are ones that not only philosophers, but scientists themselves draw. Hence, those distinctions have independent grounding in the history of science. Second, and most importantly, those distinctions are a useful addition to the philosophical vocabulary. In particular, the claim that arguments like Heisenberg's, Galton's or Schelling's draw upon similarities in non-causal features is helpful for understanding how those analogical arguments work. One specific feature of those arguments that I mean to highlight may be referred to as their larger resilience. This property can be illustrated through a comparison. First, recall Galton's example. In that case, we know from the start that the 'low level' details of the quincunx are different than those of heredity: the specific stories of how, owing to the force of gravity, pellets fall from the top of the quincunx and reach the vertical compartments at the bottom has very little in common with heredity processes in real-world populations. But now compare how the discovery of a 'low level' dissimilarity in the way the human immune system works

\footnotetext{
16 This is, indeed, what Sugden (2000) does in the case of Schelling's checkerboard argument. A similar attitude may be suggested by Hesse's (1963) remark that some factor only needs to be a cause "in some scientifically acceptable sense" (72) in order to play a role in inductive argument. (Elsewhere, though, Hesse is clear that the intended sense of 'causal connection' is the empirical kind: see, for instance, the quote in fn.2.).
} 
instead weakens an argument from the effects of a drug on mice to similar effects on humans.

The lesson I mean to draw is the following: when an argument is based on noncausal analogy, the connections between the model's properties that the argument exploits hold quite regardless of the specific low level, mechanical details of the model; as a result, the strength of that argument is comparatively more resilient to differences in low level detail detectable between model and target. Thus, the fact that the vertical relations in Galton's analogy are non-causal arguably explains why the dissimilarities in low level details between the quincunx and heredity processes are less important to the overall plausibility of Galton's conclusions than they would be if the vertical relations were causal. ${ }^{17}$ Similarly for the other examples of section two: the fact that Schelling's analogy is non-causal, for instance, arguably explains why his argument is especially resilient to known differences between the moving of dimes and pennies on a checkerboard and the moving of people in a city; the belief that $\mathrm{SU}(2)$ symmetries transcend the specific causal profiles of electrons is also likely among the considerations that led Heisenberg to postulate mathematically identical symmetries in the neutron-proton pairs despite the obvious differences between those pairs as well as between the spaces in which the symmetries are defined. Reserving a special category for inferences from non-causal analogy is therefore important for understanding how they work from an epistemological perspective. $^{18}$

To summarize the two subsections above, attempts at rescuing Hesse's classification of arguments from scientific analogies are not promising. Recognizing arguments from non-causal analogies in science as a distinctive form of scientific inference stands out as the most reasonable option. In the next section, I will complete the defense of the claim that arguments from non-causal analogies are a novel class of inferences yet unrecognized in the philosophical literature.

\section{Why novel?}

So far, my concern has been to show that the case-studies of section two refute Hesse's original classification of argument from scientific analogies. However, the case for the view that the arguments of section two constitute a yet unrecognized class of analogical inferences in science is not complete unless we can show that the same examples do not find a place in other taxonomies available in the recent literature. In what follows, I will consider this question with respect to the classification proposed by Bartha $(2009,2019)$ - arguably the most authoritative of its kind. The taxonomy starts off from the distinction between two directions of analogical

\footnotetext{
17 On the idea of resiliency to low level detail, see also the discussion of "minimal models" in Batterman and Rice (2014). Importantly, my notion of resiliency is linked to inductive strength and not to explanatory power.

${ }^{18}$ Cf. also Bartha (2009): "the strength of [the vertical relation] strongly influences the plausibility" (99).
} 
reasoning: either from a similarity in causes (or assumptions) to a similarity in the effects (or consequences), or vice versa. In the former case, Bartha refers to the analogical argument as a "predictive" one; in the latter case, as an "abductive" one (2009:99). ${ }^{19}$ Bartha then further distinguishes analogical arguments on the basis of the relation that obtains between the causes (or assumptions) and the effects (or consequences): this can be either a "deductive" relation (i.e., entailment) or an "inductive" one (such as causal or probabilistic) (2009:99). The result is an elaborate taxonomy of analogical arguments employed in scientific investigation.

An important feature of Bartha's taxonomy is that practically no significance is attached to the nature of the horizontal relations between the terms of source and target. Indeed, on Bartha's (2009:44) view, the nature of the horizontal relations makes little to no difference to the strength of an analogical argument vis-à-vis the nature of the vertical relation involved. Unlike Hesse, then, Bartha does not distinguish between 'material' and 'formal analogy'. For him, 'formal analogy' is simply a marker that "we can abstract a common mathematical form [between source and target]... regardless of whether this relates laws or descriptions of empirical phenomena" (210). At least in principle, then, any analogical argument in his taxonomy can be based on 'formal analogy'. In what follows, I will not be concerned with whether Bartha is right to neglect the nature of the horizontal relations in the classification. My aim will be solely to show that his taxonomy is insufficiently precise to account for the case-studies of section two.

Because the relation between the observed and the predicted resemblances in the examples of section two is deductive, there are only two classes of analogical arguments in Bartha's taxonomy to consider. On the one hand, Bartha countenances "mathematical" analogical arguments (2009: 108), which he understands as predictive analogical arguments (108) inferring the probability of a similarity in some consequence from a similarity in assumptions, where the assumptions and the consequences are linked by a relation of entailment. This might seem like the right category for arguments like Heisenberg's, Galton's or Schelling's, but it is not. The main issue is that the case-studies of section two are not instances of 'predictive' arguments: the reasoning in each case goes from a similarity in consequences (e.g., statistical identity) to a similarity in assumptions (e.g., the similar 'setup' of the quincunx and heredity) and not vice versa. Therefore, Bartha's class of "mathematical analogies" is an inadequate categorization.

On the other hand, Bartha countenances "abductive" analogical arguments (2009:123), in which "some observable result $\mathrm{E}$ is mathematically derived from a hypothesis Q together with other assumptions” (123). As Bartha points out, many of these abductive analogies are also 'formal analogies' in the liberal sense that there exists a mathematical resemblance between the hypothesis Q postulated in the target and the corresponding hypothesis $\mathrm{Q}^{*}$ that is well-confirmed in

\footnotetext{
19 Bartha (2009:99) also countenances two limit cases: 'bi-directional' (or 'functional') analogies where the reasoning can go both ways and 'correlative' where the direction of the argument is unknown. These further categories can be safely set aside here as they do not affect the problem of classifying non-causal analogies.
} 
the source. His examples are Priestley's argument to the inverse square electrostatic law from the analogy with the law of gravity and Stokes' argument for the harmonic law of electrons from the analogy between spectral lines and harmonic overtones. In both cases, a similarity in some effects (e.g., an observed electrostatic 'null-effect' in Priestley's experiment with charged spherical shells, which resembles the absence of gravitational attractions inside a hollow sphere of uniform density) is used to infer to a similarity in the laws that entail those effects. Hence, we have an abductive analogical argument where source and target are linked by formal analogy.

However, the case-studies of section two do not satisfy this description either. This is because, in the example that Bartha considers, the vertical relations between the observable result $\mathrm{E}$ and the hypothesis $\mathrm{Q}$ are entailment relations mediated by causal laws. The inverse-square electrostatic law in Priestley's example, for instance, is a causal law because it mediates tendencies to co-occur between given causes (e.g., charges) and their effects (e.g., motions). ${ }^{20}$ Conversely, in the examples of section two there are no causal laws involved: for instance, the vertical relation of entailment between the exchangeability properties of neutron-proton pairs and their SU(2) symmetry is mediated by a physical constraint (not an ordinary causal law); the vertical relation of entailment between the quincunx's setup and its tendency to replicate statistical identity, by a theorem of statistics. Since I have already stressed the importance of the causal vs. non-causal distinction in section three, I will not repeat my arguments here. ${ }^{21}$

The upshot is that Bartha's taxonomy needs refinement: the arguments of section two are a novel kind of abductive analogical argument (as well as a novel kind of arguments from 'formal analogy' in Bartha's sense). The class of abductive analogical arguments must therefore be further partitioned into 'causal' and 'non-causal', depending on whether the vertical relation in the analogy is mediated by an ordinary causal law or by some other principle. ${ }^{22}$ Once again, then, an entirely new class must be postulated in order to account for the arguments of section two.

\footnotetext{
${ }^{20}$ As such, Priestley's and Stokes' arguments fall within the category of arguments from 'material analogy' in Hesse's classification, despite the fact that the relation of the observed to the predicted resemblances is entailment. See also fn. 9 on the liberality of the notion of 'causal relation' assumed in this paper.

21 The distinction should be welcomed by Bartha, as he insists that "to understand individual analogical arguments, we should focus... on clarifying the exact nature of the relations within the source domain that are to be transferred to the target domain" (2009:149). My proposal is in the spirit of Bartha's methodology. It is also worth noting that, at least intuitively, arguments from non-causal analogies require even less similarities than inferences like Priestley's or Stokes's: the differences between, say, the quincunx machine and biological populations are much more obvious than those between gravitational and electrostatic attractions. Once again, this suggests that arguments from non-causal analogy possess augmented resilience to low-level detail.

${ }^{22}$ It is an interesting question if there are instances of predictive non-causal analogical arguments in the history of science as. All of the case-studies of section two are as abductive arguments in Bartha's classification.
} 


\section{Why important?}

In the previous sections, I have argued that there exists a category of arguments from scientific analogy that is not countenanced in authoritative classifications by Hesse (1963) and Bartha (2009). The case-studies of section two enrich our understanding of the kinds of inductive inferences that are employed in science. In particular, I have argued that inferences from non-causal analogy identify vertical relations that are modally more robust than causation; partly as the result of that, they are equipped with a characteristic form of resilience to 'low level' differences that is not shared by analogical arguments of the more ordinary 'causal' variety - at least not to the same degree. In this final section, I will briefly remark on how the recognition of non-causal analogies might be important to current debates in epistemology. My aim - it is worth clarifying - is not so much to establish any specific conclusion, but to indicate ways in which the discussion of the previous sections may play a role in advancing the contemporary debate.

It is helpful to distinguish in this context between broadly 'descriptive' and 'normative' issues pertaining to analogical reasoning in science. The former have to do with understanding the actual inferential practice adopted in scientific context and the different judgments of strength that scientists attach to analogical arguments. The latter concern specifically the justification that scientists might have for the inferential practice and for evaluating some analogical arguments as stronger or weaker than others. Let me clarify how the case-studies of section two are important for current debates about both descriptive and normative matters.

Descriptive Issues A notable feature of the arguments of section two is that, while taxonomically novel, they are plausibly subject to the same criteria of evaluation that have been proposed for analogies of more ordinary varieties. In particular, two standard generic criteria (Hesse, 1963:58; Bartha, 2009:103) plausibly apply to arguments from non-causal analogy:

(1) There must be relevant similarities between model and target;

(2) There must be no critical differences between model and target.

The rationale for these two criteria is fairly obvious. Arguments from non-causal analogy are strengthened by relevant similarities: for instance, by the resemblance between statistical identity in the quincunx and in population biology. This resemblance is 'relevant' because it is entailed by the predicted similarity (with respect to instantiating the reversion law) that figures in the analogical argument's conclusion. The absence of 'critical' differences, i.e., differences that directly and in the first instance affect the tenability of the conclusion, is also crucial to the plausibility of non-causal analogical arguments: Galton's argument would be severely weakened, for instance, if statistical identity were to obtain in the quincunx only in a limited number of trials, unlike the systematicity with which the same phenomenon occurs in the population data. 
In addition to responding to the same general evaluative criteria defended by Hesse (1963) and Bartha (2009), arguments from non-causal analogy are plausibly subject to some of the same specific criteria offered in those contributions. In particular, three criteria offered by Bartha (2009) for abductive analogies, namely "the validity of the [posited entailment relations]" and "the reasonableness of the additional assumptions" and the absence of "multiple analogies" (129) are all immediately transferable to the case-studies of section two. It seems plausible, for instance, that Galton's argument would lose much of its strength absent a valid connection between the presence of reversion and the instantiation of statistical identity. Hence, both the validity of the entailment and the reasonableness of the assumptions that lead (deductively) from the presence of reversion to statistical identity are important. An argument such as Schelling's would also be significantly weakened by the existence of an alternative model which relied on empirical evidence to suggest that segregation patterns in a given target city possess a historical (as opposed to a mathematical) explanation. The latter can be seen as a case in which the presence of an alternative model weakens the analogical argument ("multiple analogies").

The fact that arguments from non-causal analogy are taxonomically novel and, at least plausibly, evaluatively continuous with known categories of analogical arguments in science puts pressure on a recent contention by Norton (2020) regarding the value of descriptive projects about analogical reasoning in science. According to Norton, no "formal schema" (13) of the kind offered by Hesse (1963) and Bartha (2009) for classifying and evaluating analogical arguments in science is ever likely to succeed as a descriptive theory, partly because "there are always new instances to be accommodated and a need for a schema that fit more closely" (2). Norton's term 'always' is somewhat overstated, since we are dealing with finitely many examples. ${ }^{23}$ But another problem with his contention, which may be well-illustrated by non-causal analogies, is that taxonomical novelty does not necessarily imply differences in evaluative criteria. Indeed, if the brief considerations provided above are correct, we have a clear illustration of how "new instances [of analogies] to be accommodated" (Norton, 2020:2) does not imply "the need for a schema that fit more closely" (2), that is, a revision to the known evaluative standards. ${ }^{24}$

Normative Issues According to a popular epistemological view, which Steiner (1998) takes to be constitutive of "philosophical naturalism" (18), our justification for inductive reasoning in science depends heavily upon the use of truly scientific

\footnotetext{
${ }^{23}$ Cf. J. L. Austin (1970) on jumping too quickly to the infinite complexity of a given subject matter: "Philosophers will do this when they have listed as many, let us say, as seventeen [examples]" (234).

24 A different argument from Norton (2020) stems from the vagueness of the formal schemata offered by Hesse (1963) and Bartha (2009). For instance, Norton (2020:5) complains that Hesse's "causal relation" is vague. However, for Hesse (1963) the main task of the formal schema is not so much to settle, but to help articulate, scientific disagreements about the plausibility assigned to various analogical arguments in science. This pluralist (arguably Carnapian) attitude emerges often: see, for instance, her discussion of analogies in pre- and post-Darwinian biology in (1963:84-85). Bartha's (2009) articulation model is similarly intended to model an ideal exchange between a proponent and an opponent which share various implicit background assumptions. The vagueness can therefore be a virtue and not a problem for their respective evaluative schemata.
} 
classification (or 'natural kinds') to describe known phenomena. For instance, from the fact that platinum and osmium conduct heat, one may plausibly conclude that rhodium also likely conducts heat. This is partly because we have correctly identified platinum, osmium and rhodium as belonging to the natural kind of metals, making it possible to draw justified inductive inferences about yet unobserved properties of elements belonging to the same kind. Conversely, from the fact that, until 1980, every US President elected in a year divisible by four died while in office one does not plausibly conclude that the 1980 US President also died while in office. ${ }^{25}$ This is partly because 'being a US President elected in a year divisible by four' is not a plausible candidate for a natural kind.

When we ask the further question of what makes a given cluster of entities or properties a 'natural kind', a popular answer in contemporary epistemology is that 'naturalness' results from a given kind's being anchored to the world's objective network of causal relations. On the prominent version of this answer defended by Boyd (1991), a 'natural kind' in science is a "cluster" (Boyd, 1991:127) whose members are united by resemblances in specific causal/ mechanical features. For instance, 'being a metal' forms a natural kind because of the resemblances in causal features that individuals in that cluster possess: e.g., conductivity. Conversely, 'being elected US President in a year divisible by four' is not similarly well-anchored to the world's objective network of causal relations, since possessing such a property neither causes nor explains much in the life and actions of past US Presidents.

What is distinctive about analogical inferences of the non-causal kind, however, is that they abstract away from the specific causal details of the model (for instance, the mechanical features of the quincunx apparatus) to project potential uniformities at some distinctly non-causal level. As exemplified by shot-dropping and heredity in population biology, there are little to no resemblances in causal features among the individuals instantiating the property 'being apt to produce statistical identity outcomes'. If it is true that non-causal analogies possess a genuine inductive use in science, then, the popular view according to which inductive inferences are justified only when the scientific classifications they rely upon track the objective network of causal relations may be in need of revision. A plausible suggestion is to contend that not all of the 'natural kinds' that science is in the business of discovering are identifiable causally: some of them may be, in an important sense, 'mathematical'. ${ }^{26}$ That is to say, corresponding to a broadening of the notion of 'inference from material analogy', a broadening of the notion of 'natural kind' may be required. A full assessment of this challenge to the received view about natural kinds and its relation to inductive inference will be the object of future work.

\footnotetext{
25 Sometimes this distinction is put in terms of the different 'projectability' of given predicates. See, e.g., Steiner's (1998) discussion, which echoes the language adopted by Goodman in his classic (1955).

26 As Steiner (1998) himself acknowledges, such a view (which he calls "weak Pythagoreanism", 1998:60) is a potential candidate for addressing his mystery of the 'applicability of mathematics', i.e., the problem of the striking predictive success of inductive inferences in particle physics based on non-causal classifications.
} 
Acknowledgements I am grateful to Marc Lange, Matt Kotzen, Alan Nelson, Ram Neta, Samantha Wakil and two anonymous referees for helpful discussions regarding the arguments of this paper. A special thanks also goes to Raphael Ginsberg, Pavel Nitchovski and the Philosophy graduate student body at UNC - I could not have pursued this research without their support and solidarity over the past year.

Funding Open access funding provided by Politecnico di Milano within the CRUI-CARE Agreement.

\section{Declarations}

Ethical approval not applicable.

\section{Conflict of Interest none.}

Informed consent not applicable.

Open Access This article is licensed under a Creative Commons Attribution 4.0 International License, which permits use, sharing, adaptation, distribution and reproduction in any medium or format, as long as you give appropriate credit to the original author(s) and the source, provide a link to the Creative Commons licence, and indicate if changes were made. The images or other third party material in this article are included in the article's Creative Commons licence, unless indicated otherwise in a credit line to the material. If material is not included in the article's Creative Commons licence and your intended use is not permitted by statutory regulation or exceeds the permitted use, you will need to obtain permission directly from the copyright holder. To view a copy of this licence, visit http://creativecommons.org/licen ses/by/4.0/.

\section{References}

Achinstein, P. (1964). Models, Analogies and Theories. Philosophy of Science, 31, 328-349.

Achinstein, P. (2013). Evidence and Method: Scientific Strategies of Isaac Newton and James Clerk Maxwell. Oxford University Press.

Austin, J. L. (1970). Philosophical Papers. Oxford University Press.

Bartha, P. (2009). By Parallel Reasoning. Oxford University Press.

Bartha, P. (2019). “Analogy and Analogical Reasoning”, in Zalta, E. (ed.) Stanford Encyclopedia of Philosophy, Spring 2019 Edition.

Batterman, R., \& Rice, C. (2014). Minimal Model Explanations. Philosophy of Science, 81(3), 349-376.

Bayler-Jones, D. (2013). Scientific Models in Philosophy of Science. University of Pittsburgh Press.

Boyd, R. (1991). Realism, Anti-Foundationalism and Enthusiasm for Natural Kinds. Philosophical Studies, 61, 127-148.

Buckley, P., \& Peat, D. (Eds.). (1996). Glimpsing Reality. Routledge.

Dardashti, R., Hartmann, S., Thébault, K., \& Winsberg, E. (2019). "Hawking Radiation and Analogue Experiments: A Bayesian Analysis", Studies in the History and Philosophy of Science Part B, 1-11.

Darrigol, O. (1988). The Quantum Electrodynamics Analogy in Early Nuclear Theory or the Roots of Yukawa's Theory. Revue D'histoire Des Sciences, 41(3), 225-297.

Fraser, D. (2019). "The Non-Miraculous Success of Formal Analogies in Quantum Theories”, in French, S. and Saatsi, J. (eds.), Scientific Realism and the Quantum, New York: Oxford University Press.

French, S. (2000). The Reasonable Effectiveness of Mathematics: Partial Structures and the Application of Group Theory to Physics. Synthese, 125, 103-120.

Fumagalli, R. (2015). "No Learning from Minimal Models", Philosophy of Science, Proceedings of the $24^{\text {th }}$ Biennial Meeting of the Philosophy of Science Association.

Galton, F. (1877). Typical Laws of Heredity. Nature, 15(5), 492-533.

Goodman, N. (1955). Fact. Harvard University Press.

Grüne-Yanoff, T. (2013). Appraising Models Non-Representationally. Philosophy of Science, 80(5), $850-861$.

Hacking, I. (1990). The Taming of Chance. Cambridge University Press. 
Heisenberg, W. (1932). Uber den Bau der Atomenkerne I. Zeitschrift Fur Physics, 77, 1-11.

Hesse, M. (1963). Models and Analogies in Science. University of Notre Dame Press.

Lange, M. (2016). Because Without Cause. Oxford University Press.

Maxwell, J. C. (1890). The Scientific Papers of J. C. Maxwell, Cambridge, UK: Cambridge University Press.

Nappo, F. (2020). "Learning from Non-Causal Models", Erkenntnis, published online.

Norton, J. (2020). The Material Theory of Induction, available online.

Pietsch, W. (2019). A Causal Approach to Analogy. Journal for General Philosophy of Science, 50(4), 489-520.

Pincock, C. (2012). Mathematical Models of Biological Patterns. Lessons from Hamilton's Selfish Herd. Biology and Philosophy, 27, 481-496.

Schelling, T. (1978). Micromotives and Macrobehavior. Norton.

Steiner, M. (1998). The Applicability of Mathematics as a Philosophical Problem. Harvard University Press.

Stigler, S. (2016). The Seven Pillars of Statistical Wisdom. Harvard University Press.

Sugden, R. (2000). Credible Worlds: The Status of Theoretical Models in Economics. Journal of Economic Methodology, 7, 1-31.

Publisher's note Springer Nature remains neutral with regard to jurisdictional claims in published maps and institutional affiliations. 